\title{
Antennal motor activity induced by pilocarpine in the American cockroach
}

Jiro Okada*, Yusuke Morimoto and Yoshihiro Toh

Department of Biology, Graduate School of Sciences, Kyushu University,

Fukuoka 812-8581, Japan

Running title: Antennal motor activity induced by pilocarpine in cockroaches

\section{Corresponding author}

Jiro Okada

Faculty of Environmental Studies

Nagasaki University

Nagasaki 852-8521, Japan

Phone/Fax: +81-95-819-2762

E-mail: jokada@nagasaki-u.ac.jp

\section{Footnotes}

*Present address: Faculty of Environmental Studies, Nagasaki University, Nagasaki 852-8521, Japan

Abbreviations: AM, antennal muscle; AMNv, antennal motor nerve; $\mathrm{AbS}$, abductor of the scape; AdS, adductor of the scape; LS, levator of the scape; LP, levator of the pedicel; DP, depressor of the pedicel; CI, correlation index; CPG, central pattern generator; IC, injected drug concentration; FC, final drug concentration 


\begin{abstract}
The antennal motor system was activated by the muscarinic agonist pilocarpine in the American cockroach Periplaneta americana, and its output patterns were examined both in restrained intact animals and in isolated CNS preparations. The 3-D antennal movements induced by the hemocoelic drug injection were analyzed in in vivo preparations. Pilocarpine effectively induced prolonged rhythmic movements of both antennae. The antennae tended to describe a spatially patterned trajectory, forming loops or the symbol of infinity $(\infty)$. Such spatial regularity is comparable to that during spontaneous tethered-walking. Rhythmic bursting activities of the antennal motor nerves in in vitro preparations were also elicited by bath application of pilocarpine.

Cross-correlation analyses of the bursting spike activities revealed significant couplings among certain motor units, implying the spatial regularity of the antennal trajectory. The pilocarpine-induced rhythmic activity of antennal motor nerves was effectively suppressed by the muscarinic antagonist atropine. These results indicate that the activation of the antennal motor system is mediated by muscarinic receptors.
\end{abstract}

Keywords: insect, antenna, motor system, muscarinic, pilocarpine 


\section{Introduction}

It is generally accepted that the antennae are vital sensory appendages for insects. On the surface of the antenna, there are morphologically and functionally different types of sensilla that are sensitive to various chemical and physical stimuli (Schneider 1964; Altner and Prillinger 1980; Chapman 1982; Keil and Steinbrecht 1984; Steinbrecht 1984; Zacharuk 1985). The internal mechanoreceptors inside the basal segments of the antenna may also respond to various physical stimuli (Field and Matheson 1998; Yack 2004). The insect antenna therefore functions as a multimodal sensor.

Another particular feature of the insect antenna is its mobility - this may enable the animal to gain environmental information more efficiently (Staudacher et al. 2005). In fact, insects oscillate their antennae vigorously when they detect attractive odors such as sex pheromones (Rust et al. 1976; Olberg 1983; Nishiyama et al. 2007). Repetitive contacts of antennae may also occur when insects encounter obstacles or gaps (Pelletier and McLeod 1994; Dürr et al. 2001; Watson et al. 2002; Blaesing and Cruse 2004), conspecific mates (Loher and Rence 1978), physical objects to approach (Okada and Toh 2006), and tactile objects to discriminate (Martin 1965; Kevan and Lane 1985; Erber et al. 1998). These behavioral observations clearly indicate that the insect antenna is a typical example of an active sensor. Therefore, the manner in which the antennae swing in space would be a key aspect in collecting environmental information efficiently.

The antennal motor system has been examined in honeybees (Snodgrass 1956; Kloppenburg 1995), cockroaches (Guthrie and Tindall 1968; Baba and Comer 2008), locusts (Gewecke 1972; Bauer and Gewecke 1991), crickets (Honegger et al. 1990a), moths (Kloppenburg et al. 1997), and stick insects (Dürr et al. 2001), using either anatomical or physiological techniques. These studies revealed that approximately $10-20$ antennal motor neurons are located in the deutocerebrum where the primary afferents of 
antennal mechanoreceptors terminate and possibly make contact with interneurons (Rospars 1988; Homberg et al. 1989; Staudacher et al. 2005). However, with the exception of stick insects (Dürr et al. 2001) and cockroaches (Okada and Toh 2004, 2006), the voluntary movements of antennae in three-dimensional space and their importance for active sensing are rarely studied. Moreover, there are few physiological studies on insect antennae with reference to active sensing. A possible explanation for this deficiency is that the spontaneous antennal movement itself would be considerably suppressed by invasive treatments prior to physiological experiments.

It is well known that both nicotinic and muscarinic receptors exist in the CNS in various insect species (Sattelle 1985; Breer and Sattelle 1987; Osborne 1996). In addition, mixed property receptors that can be activated by both nicotinic and muscarinic agonists have also been reported (David and Pitman 1993). Some muscarinic agonists exert excitatory effects on various motor systems in arthropods. For example, in crustaceans, muscarinic agonists induce rhythmic motor activities in the locomotor system (Chrachri and Clarac 1990; Braun and Mulloney 1993) and the stomatogastric system (Marder and Paupardin-Tritsch 1978; Elson and Selverston 1992; Bal et al. 1994). In insects, similar muscarinic agents activate a variety of motor systems involved in feeding (Gorczyca et al. 1991; Rast and Bräunig 1997), walking (Ryckebusch and Laurent 1993; Büschges et al. 1995; Johnston and Levine 1996; Libersat et al. 1999), and fight (Buhl et al. 2008). Since these excitatory effects on arthropod motor systems were observable even in isolated CNS preparations, the drugs probably activated the central pattern generators (CPGs).

Here, we first demonstrated that the muscarinic agonist pilocarpine induces rhythmic activity of the antennal motor system in the American cockroach Periplaneta americana in both in vivo and in vitro preparations. The spatiotemporal aspects of pilocarpine-induced activity of the antennal motor system were investigated in comparison with natural movements in intact cockroaches. 


\section{Materials and methods}

Animals

Adult male cockroaches (Periplaneta americana, L.) were obtained from a laboratory colony that was maintained at $27^{\circ} \mathrm{C}$ on a $12: 12 \mathrm{LD}$ cycle.

In vivo experiments

Preparation: Spontaneous antennal movements of P. americana are generally suppressed under light conditions (Okada, personal observation). Since the in vivo experiments were necessarily conducted under near-UV conditions as described below, the compound eyes and ocelli of the animals were cauterized in order to eliminate all vision (Okada and Toh 2004). To allow for recovery from the operation, the experiments on the treated animals were initiated at least $24 \mathrm{~h}$ later. The animal was restrained in an acrylic holder, and the head capsule was glued to the holder with beeswax, leaving only the antennae freely moveable (Fig. 1a). The holder was attached to a clamp stand in an appropriate position. In order to capture the antennal motion in reference to the head capsule, several spots were marked on the cuticles of both antennae and on the head capsule with acrylic fluorescent paint (Lumino Sign No.5; Sinloihi, Kamakura, Japan) - two spots at the centers of the ocelli, one on the center of the horizontal boundary between the frons and the clypeus, and two on both the flagella approximately $2 \mathrm{~mm}$ from their proximal ends (Fig. 1b, c). A small opening (approximately $0.5 \mathrm{~mm}$ in diameter) was punched with an insect pin into the cuticle of the vertex of the head capsule for hemocoelic injection of drug solutions into the head capsule. Pilocarpine hydrochloride (Sigma-Aldrich, St. Louis, MO) dissolved in distilled water were injected by a microsyringe through a flexible polyethylene tube (outer diameter of the tip, approximately $0.5 \mathrm{~mm}$ ). The injected volume was $2 \mu \mathrm{l}$ in all experiments.

Apparatus: The 3-D antennal movements were recorded by a Machine Vision System 
(CV-2000; Keyence, Osaka, Japan) which is equipped with a pair of stereo CCD cameras and detects user-defined features in images (Fig. 1a). The two cameras were positioned at arbitrary angles in front of the head. A near-UV fluorescent lamp with a peak wavelength of $352 \mathrm{~nm}$ (FL20S BLB-A; Toshiba, Japan) was set above the specimen in order to highlight the marker spots on the head capsule and the antennae. The Machine Vision System transformed monochromatic images into binary data at a user-defined critical level of brightness and registered 2-D coordinates of the center of mass for each maker spot (resolution, $512 \times 480$; horizontal $\times$ vertical $)($ Fig. 1 b, c). The sampling rate was set at $20 \mathrm{~Hz}$. The spatial resolution achieved was estimated to be approximately $60-80 \mu \mathrm{m}$. The data were transferred online to a PC and stored in a text format.

Recording: The antennal position was recorded for at least 30 min immediately after injecting a drug. Tests for each drug concentration were repeated 5-7 times for 3-7 animals. In case of repetitive tests on a single animal (a maximum of three times), a time period of at least $24 \mathrm{~h}$ was interposed for recovery from the previous test.

Analysis: Transformation of 2-D coordinates of the recorded marker spots into 3-D coordinates was achieved by a direct linear transformation (DLT) algorithm (Abdel-Aziz and Karara 1971) installed in a 3-D motion analysis software (DippMotion XD; Ditect, Tokyo, Japan). The calibration process was conducted with a specific acrylic cube of known size $(10 \times 10 \times 10 \mathrm{~mm})$.

The antennal position with reference to the head capsule was represented as described in a previous study (modified from Nishiyama et al. 2007). Briefly, the rightand left-handed 3-D coordinates of the XYZ axes were employed to represent the positions of the marker spots on the right and left flagella, respectively (Fig. 1d). The origin of the coordinate system was set at the center of each antennal socket. The XZ plane was parallel to the plane formed by the three head spots. The horizontal deflections of the antennae were defined as the azimuth, and the vertical deflections were defined as 
the latitude of the flagellum with respect to the origin.

Spectral analyses of rhythmic antennal movements were performed by applying the fast Fourier transformation (FFT) function of an add-on software (Kaiseki Tool; Kyowa Electronic Instruments, Tokyo, Japan) to Microsoft Excel. Horizontal or vertical positions of the antennae were sampled for $51.2 \mathrm{~s}$ (1024 data points), and processed by the FFT function. Cross-correlation analyses were performed between the right and left antennae on the horizontal or vertical components. We calculated the correlation coefficients for appropriate pairs of the time series (sampling time, $51.2 \mathrm{~s}, 1024$ data points) over a range of $\pm 5 \mathrm{~s}$, which corresponded to time lags of $\geq 100$ data points, by using the Kaiseki Tool. The correlation index (CI) was defined as the correlation coefficient (R) at zero time lag where prominent peaks were observed in most cases (cf. Okada and Toh 2004). The critical value with the significant level at 0.05 was determined as $\mathrm{R}= \pm 0.0613$ according to the number of data points $(\mathrm{n}=1024)$. Statistical significances of the CIs were examined by the one-sample $t$-test in comparison with their nearer critical values ( $\mathrm{R}= \pm 0.0613)$, and they were judged to be statistically significant when $\mathrm{P}<0.05$.

The efficacy of the injected drugs in various concentrations was evaluated by the total angular displacement of the vertical components of both the antennae for $20 \mathrm{~min}$ after the drug injection. The data were obtained from five to seven tests for each dose. In the control experiments $(\mathrm{n}=5)$, the same volume $(2 \mu 1)$ of physiological saline for $P$. americana (van Asperen and van Esch 1956) was injected for comparison. Statistical differences between the control and test groups were surveyed by Steel's test, and they were considered significant when $\mathrm{P}<0.05$.

The final concentrations of drugs in the body fluid were estimated according to a report that the hemolymph volume of adult $P$. americana corresponds to $19 \%$ of the body weight (Wheeler 1962). In our preliminary inspection, the body weights of 
randomly selected adult males only showed variations of $727.5 \pm 30.6 \mathrm{mg}$ (mean \pm s.d., $\mathrm{n}$ $=10)$. Thus, the final concentrations were calculated on the basis of the putative mean hemolymph volume at $138 \mu \mathrm{l}$ plus injected solution volume at $2 \mu \mathrm{l}$ (total, $140 \mu \mathrm{l})$. In the following section for in vivo experiments, both injected drug concentrations (IC) and estimated final drug concentrations in the body fluid (FC) were provided together.

In vitro experiments

Preparation: An animal was decapitated, and the brain and subesophageal ganglion were isolated from the head capsule together with peripheral antennal motor nerves and thick trachea extending to the brain (Fig. 2b). The antenna is controlled by five functionally different muscles (Fig. 2a): three extrinsic muscles spanning between the tentorium and the proximal ends of the first antennal segment (scape), and two intrinsic muscles spanning between the proximal ends of the scape and those of the second segment (pedicel). These antennal muscles (AMs) are individually innervated from antennal motor nerves (AMNvs). The nomenclature of the AMs and AMNvs according to their anatomical arrangement was provided in some reports for P. americana (e.g., Guthrie and Tindall 1968; Baba and Comer 2008). However, our preliminary study confirmed the kinematic functions of AMs by observing the effects of direct electrical stimulations to them (Okada et al. 2005). Accordingly, the three extrinsic muscles or nerves were termed the abductor of the scape (AbS), the adductor of the scape (AdS), and the levator of the scape (LS). Similarly, the two intrinsic muscles or nerves were termed the levator of the pedicel (LP) and the depressor of the pedicel (DP). The five AMNvs were dissected and severed in close proximity to their muscular targets (Fig. 2b, arrows), while maintaining their connections to the CNS. The isolated preparation was placed at the bottom of an experimental chamber ( $1.0 \mathrm{ml}$ in volume $)$ with the dorsal side up, and oxygenated physiological saline was continuously superfused at $0.8-1.6 \mathrm{ml} / \mathrm{min}$. The cut ends of the 
tracheae were kept floating on the surface of the saline pool for aeration.

Recordings: The peripheral cut ends of the AMNvs of the isolated specimen were individually introduced into the tips of suction electrodes. In the experiments on unilateral AMNvs, the efferent activities of all five nerves were measured, and in the experiments on bilateral AMNvs, those of the right and left AbSs, AdSs, and LPs were measured. The signals were recorded using a conventional multichannel extracellular amplifier (MEG-6108; Nihon Kohden, Tokyo, Japan), and a PC-based A/D converter (Micro1401; CED, Cambridge, UK). The sampling rate was $15 \mathrm{kHz}$ for each channel. Analyses: The spikes recorded from AMNvs were classified into small (S), medium (M), and large (L) units according to the relative spike size (see Results for details). Our preliminary study showed that both the M and L units were closely correlated with the contraction force of AMs (Okada et al. 2005). Thus, both the M and L units were combined together, and the time series of the multi-spike frequencies were calculated at 0.1 s intervals with the use of the Spike2 software (CED; Cambridge, UK). However, with this method, the concern that the selected spikes were derived not only from the excitatory motoneurons but also the common inhibitor (CI) neurons or the dorsal unpaired median (DUM) neurons still existed (cf., Honegger et al. 1990a, 1990b; Bräunig et al. 1990; Allgäuer and Honegger 1993). We therefore excluded small spike units recorded simultaneously in multiple channels from the present analyses because they were possibly from the CI neurons. Regarding the DUM neurons, their existence could be ignored because no simultaneous spike was detected between bilateral AMNvs.

Cross-correlation analyses were performed on bilateral or unilateral pairs of AMNv activities. Correlation coefficients and correlation indexes (CIs) were calculated for appropriate pairs of the time courses of AMNv spike frequencies (sampling time, 102.4 s, i.e., 1024 data points) over a range of \pm 10 s (lags of $\geq 100$ data points) as described above. FFTs were also performed on the same data used for the 
cross-correlation analyses, with the use of the Kaiseki Tool software.

\section{Results}

Effects of pilocarpine on in vivo preparations

Prior to the application of drugs, the restrained animals were basically motionless. Typically, discontinuous antennal movements for less than 1 min were occasionally observed several times an hour. Figure 3 shows typical examples of antennal movements after the injections of saline and pilocarpine. In the control experiment, the saline-injected animal remained basically motionless. The muscarinic receptor agonist pilocarpine effectively induced continuous activity of the antennae. Rhythmic movements with large amplitudes were initiated immediately after the injection within several seconds, and they continued for up to several hours.

The dose-response relationship was examined for pilocarpine by comparing the total angular displacements of both antennae for a constant period of time (Fig. 4). Statistical analyses revealed that pilocarpine is effective only within narrow ranges of drug concentrations-from 10 to $40 \mathrm{mM}$ in IC and from 140 to $570 \mu \mathrm{M}$ in FC. The threshold concentration was estimated to be 1-10 mM IC and 14-140 $\mu \mathrm{M}$ FC. No conspicuous difference in antennal activity was observed within the effective dosages. Excessive application of pilocarpine resulted in the collapse of the rhythmicity shortly after its initiation.

We observed the behavioral responses to drug injections in free-moving animals. Immediately after the injection of pilocarpine, the animal tended to walk slowly with oscillating its antennae. However, the locomotor response soon ceased, and only the vigorous antennal movement remained. All animals injected survived for at least a few days following drug applications. 
Spatiotemporal characteristics of pilocarpine-induced antennal movements in in vivo preparations

When the pilocarpine-induced movements were represented in a 2-D coordinate of the horizontal and vertical angular positions, the trajectories varied in their spatial regularity. Figure 5 shows typical examples of spatially random and regular trajectories induced by pilocarpine at $10 \mathrm{mM} \mathrm{IC}$ and $140 \mu \mathrm{M}$ FC. The random trajectory was frequently observed within several minutes after the injection (Fig. 5a); then, it gradually changed to the spatially regular trajectory that lasted for up to a few hours (Fig. 5c). The antennal trajectories were randomly sampled at various time points to survey their spatial regularities in 43 samples from 23 animals that exhibited steady continuous movements of both antennae. In each sample, both the right and left antennae were subjected to the following analyses (data length, $51.2 \mathrm{~s}$ ). As judged by the experimenters, regularity was observed in 29 and 32 samples in the right (R) and left (L) antennae, respectively. The regular trajectories mainly consisted of two elements describing the symbol of infinity $(\infty)$ and a simple loop (Fig. 5c, e). During the infinity pattern, one cycle of horizontal displacement corresponded to two cycles of vertical displacement (Fig. 5e, Infinity). When the trajectory changed to the loop pattern, the cycles of both components corresponded in a one-to-one manner with appropriate delays (approximately 50-150 ms in Fig. 5e, Loop). The infinity trajectories were always produced by the same sequence of antennal movements, whereas the loop trajectories almost equally included both clockwise and counterclockwise movements (Figs. 5e, 10c1). In order to characterize the temporal aspects of the horizontal and vertical components, spectral analyses were performed. In the initial phase with random trajectories, the spectral curves of both components showed a relatively weak overlap (Fig. 5b). On the other hand, in the following phase with spatially regular trajectories, clear peaks of both components 
emerged at particular frequencies in one-to-one and one-to-two relationships (Fig. 5d). Presumably, the peaks at the same frequency correspond to the loop pattern, and the peaks with the one-to-two relationship correspond to the infinity pattern. In the samples exhibiting spatial regularities, the infinity trajectory was observed in 14 out of 29 (R) and 13 out of $32(\mathrm{~L})$ cases, and the loop trajectory in 14 out of $29(\mathrm{R})$ and 18 out of 32 (L) cases. The other regular trajectories were classified into the "arch" (cf. Okada and Toh 2004) or simple vertical movement. The temporal sequence of the arch trajectories was similar to that of the infinity pattern.

In intact animals, the right and left antennae generally move in concert with each other. This was the case in the pilocarpine-induced antennal activity in in vivo specimens. In order to examine temporal couplings between both antennae quantitatively, we conducted cross-correlation analyses of their movements. Significant negative couplings were detected in the horizontal components (Fig. 6). This suggests that abduction of one antenna is significantly accompanied by adduction of the other antenna and vice versa for movement in the opposite direction. For the vertical component, a bilateral positive coupling was observed, suggesting that both antennae synchronously move in the dorsoventral plane. Bilaterally coordinated behavior between the right and left antennae was also observed in the following test. When one antenna was blocked by placing an obstacle during pilocarpine-induced activity, the blocked antenna consequently withdrew or paused, otherwise passed the obstacle. At this moment, the opposite antenna tended to mimic the response of the blocked antenna in any case (data not shown).

Antennal motor outputs induced by pilocarpine in in vitro preparations

The effects of pilocarpine on antennal motor outputs were examined in isolated CNS preparations. Bath application of pilocarpine at $100-300 \mu \mathrm{M}$ induced rhythmic spike activity of the AMNvs. This concentration range of perfusate was comparable with 
the effective FC range in in vivo experiments (Fig.4). An immediate response after its application, as it occurred in the in vivo experiments, was never observed.

Figure 7 shows a typical example of pilocarpine-induced efferent activity in unilateral AMNvs. Prior to its application, the AMNvs exhibited spontaneous tonic spikes (Fig. 7a). Bath application of pilocarpine $(150 \mu \mathrm{M})$ gradually changed their discharge pattern to rhythmic bursts (Fig. 7b). Clear antiphase and inphase relationships were observed in the antagonistic pairs (LP vs. DP, LS vs. DP, and AbS vs. AdS) and agonistic pairs (LS vs. LP), respectively. The analyses of spike trains demonstrated that each muscle would be innervated by four to six motor neurons. Some spike units may originate from common neurons innervating multiple muscles. For example, unit 2 in Figure $7 \mathrm{~b}$ was the only spike that appeared in all five AMNvs. In fact, it is known that all or a part of unilateral antennal muscles may receive inhibitory innervation from the single CI neuron (cf. Honegger et al. 1990a, 1990b; Bauer and Gewecke 1991; Dürr et al. 2001; Baba and Comer 2008).

The effect of the muscarinic receptor antagonist atropine on pilocarpine-induced AMNv activity was examined (Fig. 8). The rhythmic activity of DP caused by pilocarpine at $150 \mu \mathrm{M}$ was effectively blocked by the additional application of atropine at $300 \mu \mathrm{M}$. After a wash with pilocarpine solution $(150 \mu \mathrm{M})$, the rhythmic activity was restored to its previous level. The same results were simultaneously obtained for the other AMNvs in this example (data not shown). This suggests that the induction of antennal movements by bath applied pilocarpine is mediated by muscarinic receptors in the CNS.

Temporal aspects of pilocarpine-induced AMNv activity

Spectral analyses were applied to pilocarpine-induced efferent activities of the unilateral AMNvs in order to characterize their rhythmicities. Figure 9 shows two representative cases of FFT spectra in randomly sampled 85 tests from 12 animals. When 
AMNvs are classified according to their kinematic functions, AbS and AdS belong to the horizontal group, and LS, LP, and DP to the vertical group. The FFT spectra belonging to either the same horizontal or vertical group closely resembled each other (Fig. 9a). In contrast, the relationships between the horizontal and vertical groups were rather obscure in most cases. The frequencies of all AMNv activities were considerably lower compared with those measured during in vivo experiments (see Fig. 5b).

Another example was a relatively minor but remarkable spectral pattern in which clear temporal relationships were observed between the horizontal and vertical groups (Fig. 9b). The horizontal motor units had conspicuous power peaks at approximately 0.16 and $0.35 \mathrm{~Hz}$. In contrast, the vertical motor units had single peaks at approximately $0.35 \mathrm{~Hz}$. Thus, one-to-one and one-to-two relationships appeared between the horizontal and vertical groups in this example, similar to the in vivo preparations (see Fig. 5d). Such relationships imply spatiotemporal regularity in antennal movement.

Unilateral and bilateral couplings in pilocarpine-induced AMNv activity

Cross-correlation analyses were performed to examine functional couplings between the antennal motor units (Fig. 10). The correlation indexes (CIs) were calculated for unilateral and bilateral pairs of AMNvs. In the unilateral AMNvs (Fig. 10a), the analyses of randomly selected 69 samples from 12 animals detected strong couplings in the agonistic and antagonistic pairs, i.e., AbS vs. AdS, LS vs. LP, LS vs. DP, and LP vs. DP. This result was very reasonable considering their functional relationships. In contrast, significant correlates between the horizontal and vertical motor units were detected only in two pairs (AbS vs. DP, and AdS vs. DP) out of six, which were relatively weak inphase couplings. These functional correlates may contribute to at least particular phases in the spatially regular patterns (infinities and loops) frequently observed in in vivo experiments (Fig. 10, c1) 
The same analyses were performed on the bilateral antennal motor units for AbSs, AdSs, and LPs (Fig. 10b). In the three bilateral motor unit pairs, we could not observe any common spike unit. The AMNv activities were considerably different bilaterally in more than half of samples. For example, AMNvs on one side expressed clear rhythmicities, but the AMNvs on the other side exhibited only slow tonic activity. The bilateral coupling is merely weak in in vitro conditions, probably due to the lack of mechanosensory feedback (see Discussion). We nevertheless conducted cross-correlation analyses in 42 samples from five animals that showed rhythmic bursts on both sides. The analyses resulted in subtle but significant bilateral couplings in AbSs and LPs in an antiphase and inphase manner suggesting that simultaneous abduction of both antennae is less frequent, whereas simultaneous levation frequently occurs, respectively (Fig. 10, c2).

\section{Discussion}

Muscarinic activation of theantennal motor system

In the present study, the muscarinic agonist pilocarpine was found to be a potent agent to elicit persistent activity of the antennal motor system in both in vivo and in vitro preparations. In fact, the existence of muscarinic $\mathrm{ACh}$ receptor (mAChR) was reported in the brain of P. americana (Orr et al. 1991). Since the effective dose was somewhat high ( $>100 \mu \mathrm{M}$ in final concentration) to justify the ligand selectivity, the drug concentration at unknown neuronal targets should be considerably lower than that in the body fluid. This is a common pharmacological feature that is probably due to the interference of drug diffusion through the less permeable ganglionic sheath and the structural difference of the insect mAChRs from the vertebrate mAChRs (e.g., Ryckebusch and Laurent 1993;

Büschges et al. 1995; Johnston and Levine 1996). Büschges et al. (1995) reported in stick insects that threshold concentrations of bath-applied pilocarpine $(>100 \mu \mathrm{M})$ were 
diminished down to $1 \mu \mathrm{M}$ by desheathing the ganglia for induction of the fictive walking rhythmicity. The present dose-response relationship in in vivo experiments showed specific patterns (see Fig. 4), and the most effective dosage could not be judged clearly. When pilocarpine at a high concentration $(>500 \mu \mathrm{M})$ was applied to an in vitro preparation, steady rhythmic outputs of the unilateral AMNvs were soon established, but they gradually became faster, and finally terminated after unusual high-frequency discharges (data not shown). Therefore, the prolonged rhythmic activity of the antennal motor system would be elicited only by a relatively narrow concentration range of pilocarpine.

The evoked AMNv activity in in vitro experiments implies that the unilateral antennal motor units are coupled to each other even without afferent inputs. Our preliminary experiments showed that the antennal motor activity could be generated by pilocarpine in the isolated lone brain preparation although its output was weak and unstable in comparison with the present in vitro preparation (brain plus subesophageal ganglion). We therefore speculate that pilocarpine affects the putative antennal CPG presumably located in the deutocerebrum. However, the effective site of pilocarpine is thus far completely unknown.

The pilocarpine-induced bursting activities of AMNvs in in vitro preparations were considerably slower in their cycles than the antennal movements in in vivo preparations (Figs. 5d, 9b). This feature has also been described in other studies using isolated CNS preparations and it may relate to the lack of sensory feedback (e.g., Chrachri and Clarac 1990; Ryckebusch and Laurent 1993; Johnston and Levine 1996). We previously reported that the ablation of antennal mechanoreceptors (scapal hair plates) in the cockroach results in an increase of the slower component in the FFT spectrum for horizontal antennal movement (Okada et al. 2002). The afferent signals from antennal mechanoreceptors, which provide information regarding the deflections of 
antennal joints, may play a crucial role in the temporal aspects of motor pattern generation (cf. Pearson 1993). Horseman et al. (1997) reported that in crickets, the subesophageal ganglion is essential for continuous antennal movement and that the thoracic ganglia are related to fast antennal movement. Therefore, the ascending signals from the subesophageal ganglion or thoracic ganglia could also affect the temporal aspects of the antennal motor pattern in cockroaches.

Some aspects of antennal responses to pilocarpine were inconsistent between the intact and isolated preparations, such as the immediate activation of antennal movements only in in vivo preparations (Fig. 3). Although the neural mechanism for interpreting this unusual phenomenon is completely unknown, it would be due to extremely high concentration of pilocarpine (>10 $\mathrm{mM}$ in IC) inside the head capsule immediately after injection. Similar response to high concentration of pilocarpine was recently described in the flight motor system of locusts (Buhl et al. 2008). A useful and reliable method to localize the effective sites of drugs is their focal injection into particular brain areas such as the deutocerebrum. This should be applied to both in vivo and in vitro preparations in future studies.

Functional couplings among unilateral antennal motor units

The antennal trajectories induced by cholinergic agonists involved spatially regular patterns. In our previous study, spatial regularity was present during or immediately after the intended orientation (Okada and Toh 2006). We have reported three basic pattern elements, i.e., the loops, arches, and vertical deflections (Okada and Toh 2004); moreover, the existence of the infinity pattern itself has been identified in voluntary antennal movements (unpublished). Thus, spatial regularity would be a common feature in both natural and pharmacologically-created conditions.

The cross-correlation analysis applied to the in vitro data revealed significant 
couplings between the horizontal and vertical motor units in some pairs (AbS vs. DP and AdS vs. DP) (Fig. 10a). This phenomenon may contribute to at least particular phases in the spatially regular patterns frequently observed in in vivo preparations (Fig. 10c). Correlations between the rest of the pairs (Fig. 10a; AbS vs. LS, AbS vs. LP, AdS vs. LS, and AdS vs. LP) were judged to be statistically insignificant. However, the individual CI values were beyond the significant level $(|\mathrm{R}|>0.0613)$ in most cases. Therefore, the result of statistics reflects inconsistence of the coupling mode (positive/negative) instead of weakness in the coupling strength. We suspect that the mode and strength of functional couplings among the antennal motor units are flexibly changeable depending on various internal and external factors. Recent studies on the CPGs for locomotor systems have emphasized their flexibility in the output patterns rather than their stereotypy since flexibility is considered to be crucial in adaptive behaviors (Bässler and Büschges 1998; Clarac et al. 2000; Pearson 2000; Büschges 2005). In addition to these known effector systems, the present findings on the antennal motor system provide a novel insight into the adaptive control of an active sensor.

Thus far, the significances of the random and spatially regular movements of cockroach antennae are totally unknown. Krause and Dürr (2004) demonstrated in their simulation study on stick insects that antennal movement with spatiotemporal regularity is advantageous in gaining tactile information effectively with the least energy expenditure under certain conditions. A similar simulation study for the antenna of $P$. americana would provide clues for understanding the significance of spatial regularity in antennal movements.

Bilateral coupling of drug-induced antennal movements

The coupling analyses between the right and left antennae revealed significant correlations in both the horizontal and vertical components in in vivo preparations (Fig. 6). 
In fact, intact cockroaches generally oscillate both antennae in a coordinated manner: the horizontal positions basically correspond with each other, i.e., the outward movement (abduction) of one antenna is accompanied by the inward movement (adduction) of the other antenna and vice versa for movement in the opposite direction (Okada and Toh 2004). In addition, the vertical movements of both antennae are synchronized in an inphase manner particularly during walking (Okada and Toh 2004). In contrast, in the in vitro preparations, the right and left antennal motor units behaved rather independently. Similar bilateral uncoupling was reported in pilocarpine-induced activities of deafferented locomotor systems of locusts (Ryckebusch and Laurent 1993) and stick insects (Büschges et al. 1995) although such cases were very few. It is possible that the bilateral coupling of antennal motor centers is mediated largely by afferent inputs from the contralateral mechanoreceptors. However, if both sides of the AMNvs were simultaneously activated by pilocarpine, weak but significant bilateral couplings were nevertheless detected (Fig. 10b). This also suggests the existence of direct mutual interaction between both sides of the antennal motor centers.

\section{Acknowledgements}

This study was supported in part by Grants-in-Aid for Scientific Research (C) from JSPS (17570063 and 19570070) and the Yamada Science Foundation to JO. 


\section{References}

Abdel-Aziz YA, Karara HM (1971) Direct linear transformation from comparator coordinates into object space coordinates. In: Proceedings of the symposium on close-range photogrammetry. American Society of Photogrammetry, Falls Church, VA, pp. 1-18

Allgäuer C, Honegger H-W (1993) The antennal motor system of crickets: modulation of muscle contractions by a common inhibitor, DUM neurons, and proctolin. J Comp Physiol A 173: 485-494

Altner H, Prillinger L (1980) Ultrastructure of invertebrate chemo-, thermo, and hygroreceptors and its functional significance. Int Rev Cytol 67: 69-139

Baba Y, Comer CM (2008) Antennal motor system of the cockroach, Periplaneta americana. Cell Tissue Res 331: 751-762

Bal T, Nagy F, Moulins M (1994) Muscarinic modulation of a pattern-generating network: control of neuronal properties. J Neurosci 14: 3019-3035

Bässler U, Büschges A (1998) Pattern generation for stick insect walking movements - multisensory control of a locomotor program. Brain Res Rev 27: $65-88$

Bauer C, Gewecke M (1991) Motoneural control of antennal muscles in Locusta migratoria. J Insect Physiol 37: 551-562

Blaesing B, Cruse H (2004) Stick insect locomotion in a complex environment: climbing over large gaps. J Exp Biol 207: 1273-1286

Braun G, Mulloney B (1993) Cholinergic modulation of the swimmeret motor system in crayfish. J Neurophysiol 70: 2391-2398

Bräunig P, Allgäuer C, Honegger H-W (1990) Suboesophageal DUM neurones are part of the antennal motor system of locusts and crickets. Experientia 46: 259-261

Breer H, Sattelle DB (1987) Molecular properties and functions of insect acetylcholine 
receptors. J Insect Physiol 33: 771-790

Buhl E, Schildberger K, Stevenson PA (2008) A muscarinic cholinergic mechanism underlies activation of the central pattern generator for locust flight. J Exp Biol 211: $2346-2357$

Büschges A, Schmitz J, Bässler U (1995) Rhythmic patterns in the thoracic nerve cord of the stick insect induced by pilocarpine. J Exp Biol 98: 435-456

Büschges A (2005) Sensory control and organization of neural networks mediating coordination of multisegmental organs for locomotion. J Neurophysiol 93: $1127-1135$

Chapman RF (1982) Chemoreception: the significance of receptor numbers. Adv Insect Physiol 16: 247-356

Chrachri A, Clarac F (1990) Fictive locomotion in the fourth thoracic ganglion of the crayfish, Procambarus clarkii. J Neurosci 10: 707-719

Clarac F, Cattaert D, Ray DL (2000) Central control components of a 'simple' stretch reflex. Trends Neurosci 23: 199-208

David JA, Pitman RM (1993) The pharmacology of $\alpha$-bungarotoxin-resistant acetylcholine receptors on an identified cockroach motoneurone. J Comp Physiol A 172: 359-368

Dürr V, König Y, Kittmann R (2001) The antennal motor system of the stick insect Carausius morosus: anatomy and antennal movement pattern during walking. J Comp Physiol A 187: 131-144

Elson RC, Selverston A (1992) Mechanisms of gastric rhythm generation in the isolated stomatogastric ganglion of spiny lobsters: bursting pacemaker potentials, synaptic interactions, and muscarinic modulation. J Neurophysiol 68: 890-907

Erber J, Kierzek S, Sander E, Grandy K (1998) Tactile learning in the honeybee. J Comp Physiol A 183: 737-744 
Field LH, Matheson T (1998) Chordotonal organs of insects. Adv Insect Physiol 27: $1-228$

Gewecke M (1972) Bewegungsmechanismus und Gelenkrezeptoren der Antennen von Locusta migratoria. Z Morphol Tiere 71: 128-149

Gorcryca, M. G., I., Budnik, V., White, K. and Wu, C. F. (1991). Dual muscarinic and nicotinic action on a motor program in Drosophila. J. Neurobiol. 22, 391-404.

Guthrie DM, Tindall AR (1968) The Biology of the Cockroach. Edward Arnold Ltd, London

Homberg U, Christensen TA, Hildebrand JG (1989) Structure and function of the deutocerebrum in insects. Ann Rev Entomol 34: 477-501

Honegger H-W, Allgäuer C, Klepsch U, Welker J (1990a) Morphology of antennal motoneurons in the brains of two crickets, Gryllus birnaculatus and Gryllus campestris. J Comp Neurol 291: 256-268

Honegger H-W, Brunninger B, Bräunig P, Elekes K (1990b) GABA-like immunoreactivity in a common inhibitory neuron of the antennal motor system of crickets. Cell Tissue Res 260: 349-354

Horseman BG, Gebhardt MJ, Honegger H-W (1997) Involvement of the suboesophargeal and thoracic ganglia in the control of antennal movements in crickets. J Comp Physiol A 181: 195-204

Johnston RM, Levine RB (1996) Crawling motor patterns induced by pilocarpine in isolated larval nerve cords of Manduca sexta. J Neurophysiol 76: 3178-3195

Keil TA, Steinbrecht RA (1984) Mechanosensitive and olfactory sensilla of insects. In: King RC, Akai H (ed) Insect Ultrastructure Vol 2. Plenum Press, New York, pp $477-516$

Kevan P, Lane MA (1985) Flower pedal microtexture is a tactile cue for bees. Proc Natl Acad Sci USA 82: 4750-4752 
Kloppenburg P (1995) Anatomy of the antennal motoneurons in the brain of the honeybee (Apis meriifera). J Comp Neurol 363: 333-343

Kloppenburg P, Camazine SM, Sun XJ, Randolph P, Hildebrand JG (1997) Organization of the antennal motor system in the sphinx moth Manduca sexta. Cell Tissue Res 287: 425-433

Krause AF, Dürr V (2004) Tactile efficiency of insect antennae with two hinge joints. Biol Cybern 91: 168-181

Libersat F, Haspel G, Casagrand J, Fouad K (1999) Localization of the site of effect of a wasp's venom in the cockroach escape circuitry. J Comp Physiol A 184: $333-345$

Loher W, Rence B (1978) The mating behavior of Teleogryllus commodus (Walker) and its central and peripheral control. Z Tierpsychol 46: 225-259

Marder E, Paupardin-Tritsch D (1978) The pharmacological properties of some crustacean neuronal acetylcholine, $\gamma$-aminobutyric acid, and L-glutamate responses. J Physiol 280: 213-236

Martin H (1965) Leistungen des topochemischen Sinnes bei der Honigbiene. Z Vergl Physiol 50: 254-292

Nishiyama K, Okada J, Toh Y (2007) Antennal and locomotor responses to attractive and aversive odors in the searching cockroach. J Comp Physiol A 193: 963-971

Olberg RM (1983) Pheromone-triggered flip-flopping interneurons in the ventral nerve cord of the silkworm moth, Bombyx mori. J Comp Physiol 152: 297-307

Okada J, Kanamaru Y, Toh Y (2002) Mechanosensory control of antennal movement by scapal hair plates in the American cockroach. Zool Sci 19: 1201-1210

Okada J, Nishiyama K, Toh Y (2005) A kinematic study of the cockroach antenna. Zool Sci 22: 1475 (abstr)

Okada J, Toh Y (2004) Spatio-temporal patterns of antennal movements in the searching 
cockroach. J Exp Biol 207: 3693-3706

Okada J, Toh Y (2006) Active tactile sensing for localization of objects by the cockroach antenna. J Comp Physiol A 192: 715-726

Orr GL, Orr N, Hollingworth RM (1991) Distribution and pharmacological characterization of muscarinic-cholinergic receptors in the cockroach brain. Arch Insect Biochem Physiol 16: 107-122

Osborne RH (1996) Insect neurotransmission: neurotransmitters and their receptors. Pharmacol Ther 69: 117-142

Pearson KG (1993) Common principles of motor control in vertebrates and invertebrates. Ann Rev Neurosci 16: 265-97

Pearson KG (2000) Neural adaptation in the generation of rhythmic behavior. Ann Rev Physiol 62: 723-753

Pelletier Y, McLeod CD (1994) Obstacle perception by insect antennae during terrestrial locomotion. Physiol Entomol 19: 360-362

Rast GF, Bräunig P (1997) Pilocarpine-induced motor rhythms in the isolated locust subesophageal ganglion. J Exp Biol 200: 2197-2207

Rospars JP (1988) Structure and development of the insect antennodeutocerebral system. Int J Insect Morphol Embryol 17, 243-294

Rust MK, Burk T, Bell WJ (1976) Pheromone-stimulated locomotory and orientation responses in the American cockroach. Anim Behav 24: 52-67

Ryckebusch S, Laurent G (1993) Rhythmic patterns evoked by in locust leg motor neurons by the muscarinic agonist pilocarpine. J Neurophysiol 69: 1583-1595

Sattelle DB (1985) Acetylcholine receptors. In: Kerkut GA, Gilbert GI (ed)

Comprehensive insect physiology, biochemistry and pharmacology Vol 11. Pergamon Press, Oxford New York, pp 395-434

Schneider D (1964) Insect antennae. Ann Rev Entomol 8: 103-122 
Snodgrass RE (1956) Anatomy of the Honeybee. Cornell University Press, Ithaka London Staudacher E, Gebhardt MJ, Dürr V (2005) Antennal movements and mechanoreception: neurobiology of active tactile sensors. Adv Insect Physiol 32: 49-205

Steinbrecht RA (1984) Chemo-, hygro, and thermoreceptors. In: Bereiter-Hahn J, Matoltsy AG, Richards KS (ed) Biology of the Integument Vol 1. Springer-Verlag, Berlin, pp 523-553

van Asperen K, van Esch I (1956) The chemical composition of the haemolymph in Periplaneta americana with special reference to the mineral constitutions. Arch. Néerl Zool 11: 342-360

Watson JT, Ritzmann RE, Zill SN, Pollack AJ (2002) Control of obstacle climbing in the cockroach, Blaberus discoidalis. I. Kinematics. J Comp Physiol A 188: 39-53

Wheeler RE (1962) Studies on the total haemocyte count and haemolymph volume in Periplaneta americana (L.) with special reference to the last moulting cycle. J Insect Physiol 9: 223-235

Yack JE (2004) The structure and function of auditory chordotonal organs in insects. Microsc Res Tech 63:315-337

Zacharuk RY (1985) Antennae and sensilla. In: Kerkut GA, Gilbert GI (ed) Comprehensive insect physiology, biochemistry and pharmacology Vol 6. Pergamon Press, Oxford New York, pp 1-69 


\section{Figure legends}

Fig. 1. a. Setup for the in vivo experiments. Antennal movements were recorded by the Machine Vision System. b, c. Image processing in the Machine Vision System. A monochromatic original image captured by one camera $(\mathbf{b})$ is transformed into a binary image (c). Note that the center of mass of each marker spot is indicated by the cross symbol. d. Definition of antennal position (only the right antenna is shown here). The marker spots are indicated by stars. The plane including the three head spots (shaded triangle) was parallel to the $\mathrm{XZ}$ plane. The horizontal and vertical positions were defined as the azimuth and the latitude of the flagellum with respect to the origin (O) of the 3-D coordinates, respectively.

Fig. 2. Schematic diagrams of the antennal motor system. a. Anatomy of the antennal muscles (AMs) (frontal view). Active movements occur at two joints between the head and the scape and between the scape and the pedicel. The head-scape joint moves in both the horizontal and vertical planes, whereas the scape-pedicel joint moves only in the vertical plane. The scape is operated by three extrinsic muscles (AbS, AdS, and LS). The pedicel is operated by an antagonistic pair of intrinsic muscles (LP and DP). $\mathbf{b}$. Innervation of AMs from antennal motor nerves (AMNvs). The five AMs are innervated from two main antennal nerves arising from the brain (ANv3 and ANv4, indicated by thick solid lines). Each of the AbS and LS muscles consists of two masses. The open arrows indicate sites where the nerves were severed for extracellular recordings. ANv, antennal nerve; SEG, subesophageal ganglion

Fig. 3. Effects of pilocarpine on antennal movements. The vertical antennal position was monitored for $30 \mathrm{~min}$ after injection of the drug. The time at zero indicates the moment of injection of the drug solution $(2 \mu \mathrm{l})$. Saline unchanged the inactive state of antennae 
(upper trace). Pilocarpine (40 mM in IC, $570 \mu \mathrm{M}$ in FC) induced long-lasting rhythmic movement of the antennae immediately after its injection (lower trace).

Fig. 4. Dose-response relationship for pilocarpine. The ordinate indicates the total angular displacement of the vertical deflection in both antennae for $20 \mathrm{~min}$ after the drug injection. The upper and lower abscissae indicate injected drug concentration (IC) and estimated final drug concentration in the body fluid (FC), respectively. Each plot and vertical bars indicate the median and the $25^{\text {th }}$ and $75^{\text {th }}$ percentiles $(n=5-7)$. Asterisks denote significant differences from the control group (Steel's test, $\mathrm{P}<0.05$ ).

Fig. 5. Spatiotemporal aspects of pilocarpine-induced antennal movements. a, c. Typical patterns of the random (a) and regular (c) trajectories (sampling period, $20 \mathrm{~s}$ each). These two trajectories were obtained from the same test at $2 \mathrm{~min}$ and $5 \mathrm{~min}$ after the injection of pilocarpine (10 mM IC, $140 \mu \mathrm{M}$ FC). The crossing arrows indicate the orientation: M, medial; L, lateral; D, dorsal; V, ventral. Note that the spatially regular movements include the "infinity ( $\infty)$ " and "loop" patterns. b, d. Power spectra of the horizontal and vertical components in the random (b) and regular (d) trajectories (sampling period, $51.2 \mathrm{~s}$ each). The ordinate is represented by a normalized value. $\mathbf{d}$. Note that particular power peaks for the horizontal and vertical components appear at the frequencies with a one-to-one or one-to-two relationship (see arrows at $1.2 \mathrm{~Hz}$ and $2.3 \mathrm{~Hz}$ ). e. The time courses of the horizontal and vertical components for the infinity and loop trajectories. The example shows a transition from the infinity trajectories (shaded area on the left) to the loop trajectories (shaded area on the right), in which their temporal sequences are indicated by arrows in the corresponding trajectories.

Fig. 6. Temporal couplings of horizontal and vertical components between the right and 
left antennae in in vivo preparations. The vertical columns and bars indicate the mean correlation indexes (CIs) and the standard deviations, respectively $(n=44)$. The shaded region around zero shows the insignificant range of the $\mathrm{CI}$ value $(|\mathrm{R}|<0.0613)$. The asterisks represent statistically significant differences from the nearest critical values $(R=$ \pm 0.0613 ) (one-sample $t$-test, $\mathrm{P}<0.05$ ).

Fig. 7. Efferent activities of antennal motor nerves (AMNvs) induced by pilocarpine in an isolated CNS specimen. For abbreviations of AMNvs, see Fig. 2. a. Spontaneous efferent activity of AMNvs before application of the drug. The small and medium sizes of spikes ( $\mathrm{S}$ and $\mathrm{M}$ units) are tonically discharged at low frequencies. b. AMNv activities during bath application of pilocarpine $(150 \mu \mathrm{M})$. The record was obtained at $30 \mathrm{~min}$ from the initiation of drug application. The top set of five traces is an expansion of the middle set indicated by break lines. The bottom set represents the time courses of spike frequencies for $\mathrm{L}$ and $\mathrm{M}$ units in the middle set. Spike units were provided temporary numbers from 1 to 9 . The units $1,4,8$, and 9 appeared exclusively in single AMNvs, whereas the units 2 , $3,5,6$, and 7 were observed in more than two AMNvs. Note that only unit 2 participates in all five AMNvs.

Fig. 8. Inhibition of pilocarpine-induced AMNv activity by atropine. The spontaneous activity of DP (spontaneous) was changed to rhythmic bursting by application of pilocarpine at $150 \mu \mathrm{M}$ (pilocarpine). The bursting activity was considerably suppressed by an additional application of atropine at $300 \mu \mathrm{M}$ (pilocarpine + atropine), and restored again after washing with pilocarpine solution (wash). The data were derived at $34 \mathrm{~min}$ (pilocarpine), $11 \mathrm{~min}$ (pilocarpine + atropine), and $59 \mathrm{~min}$ (wash) after the initiation of each treatment. 
Fig. 9. Power spectra of AMNv activities induced by pilocarpine. a. A typical example of the FFT spectra for five AMNvs at 23 min after the application of $150 \mu \mathrm{M}$ pilocarpine. Note that the spectral patterns for the horizontal (AbS and AdS) or vertical (LS, LP, and DP) group were similar within each group. $\mathbf{b}$. A remarkable spectral pattern of the AMNvs. Conspicuous peaks for the horizontal group were observed at approximately 0.16 and $0.35 \mathrm{~Hz}$ (arrows) and those for the vertical group only at $0.35 \mathrm{~Hz}$, suggesting one-to-one and one-to-two relationships between the two groups. The data were derived at $33 \mathrm{~min}$ after the initiation of application of pilocarpine $(200 \mu \mathrm{M})$.

Fig. 10. Temporal couplings of AMNv activities. a. Unilateral couplings of the pilocarpine-induced activity in all AMNv pairs $(n=69)$. Strong couplings were always detected in the pairs within the horizontal or vertical group. In contrast, the couplings between the horizontal and vertical groups were basically weak. The inset shows the summary of the coupling pattern. The inphase and antiphase couplings are depicted by filled and open lines, respectively. Their relative strength is represented by the thickness of the lines. b. Bilateral couplings in three AMNv pairs $(n=42)$. The extent of the bilateral couplings was relatively very weak. c. 1. Possible contributions of significant unilateral couplings (AbS vs. DP, and AdS vs. DP) to the infinity and loop trajectories were indicated by thick solid and broken lines, respectively. 2 . Significant bilateral couplings in AbSs and LPs may affect the horizontally and vertically coordinated movements, respectively. 
Fig. 1
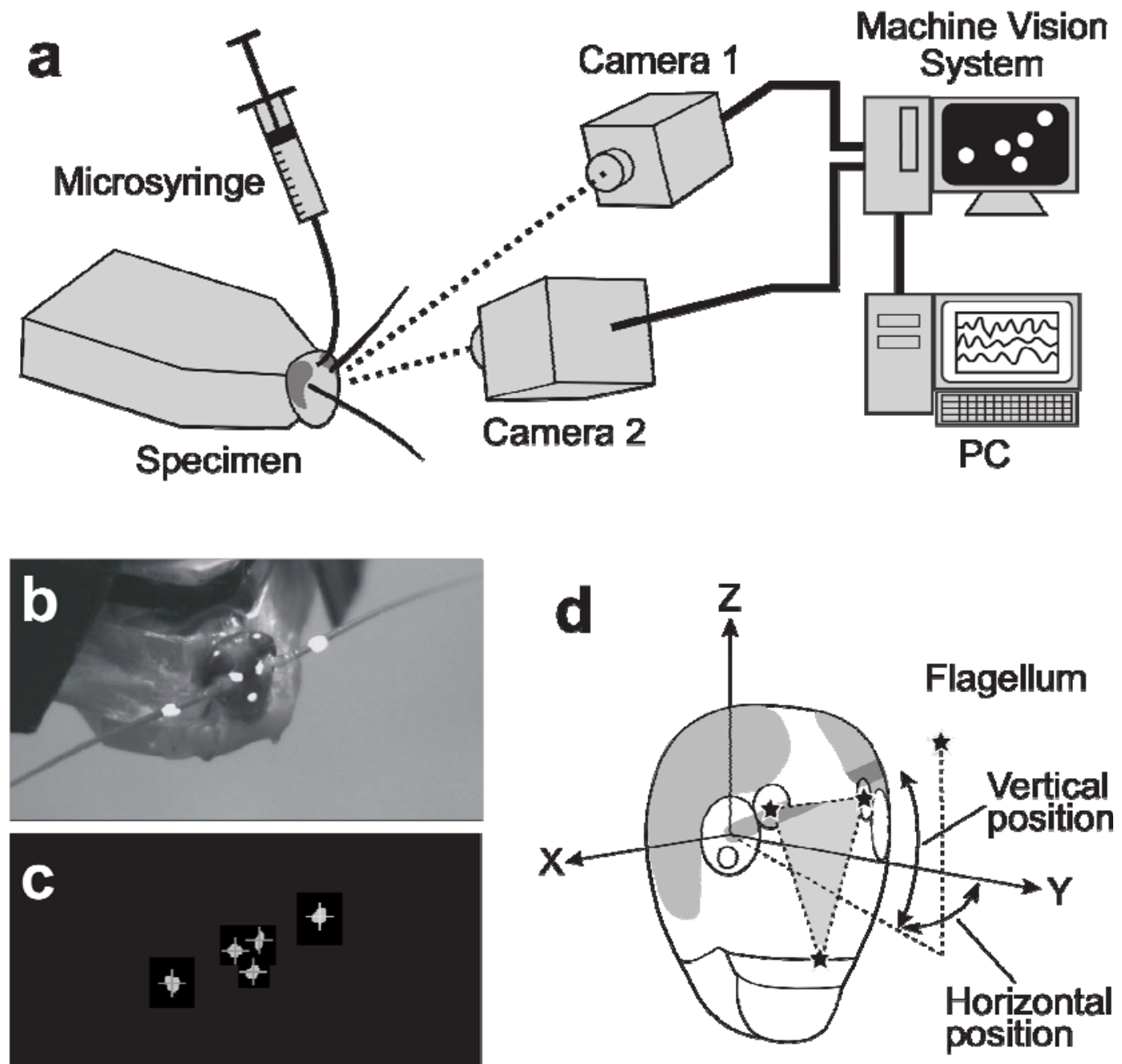
Fig. 2
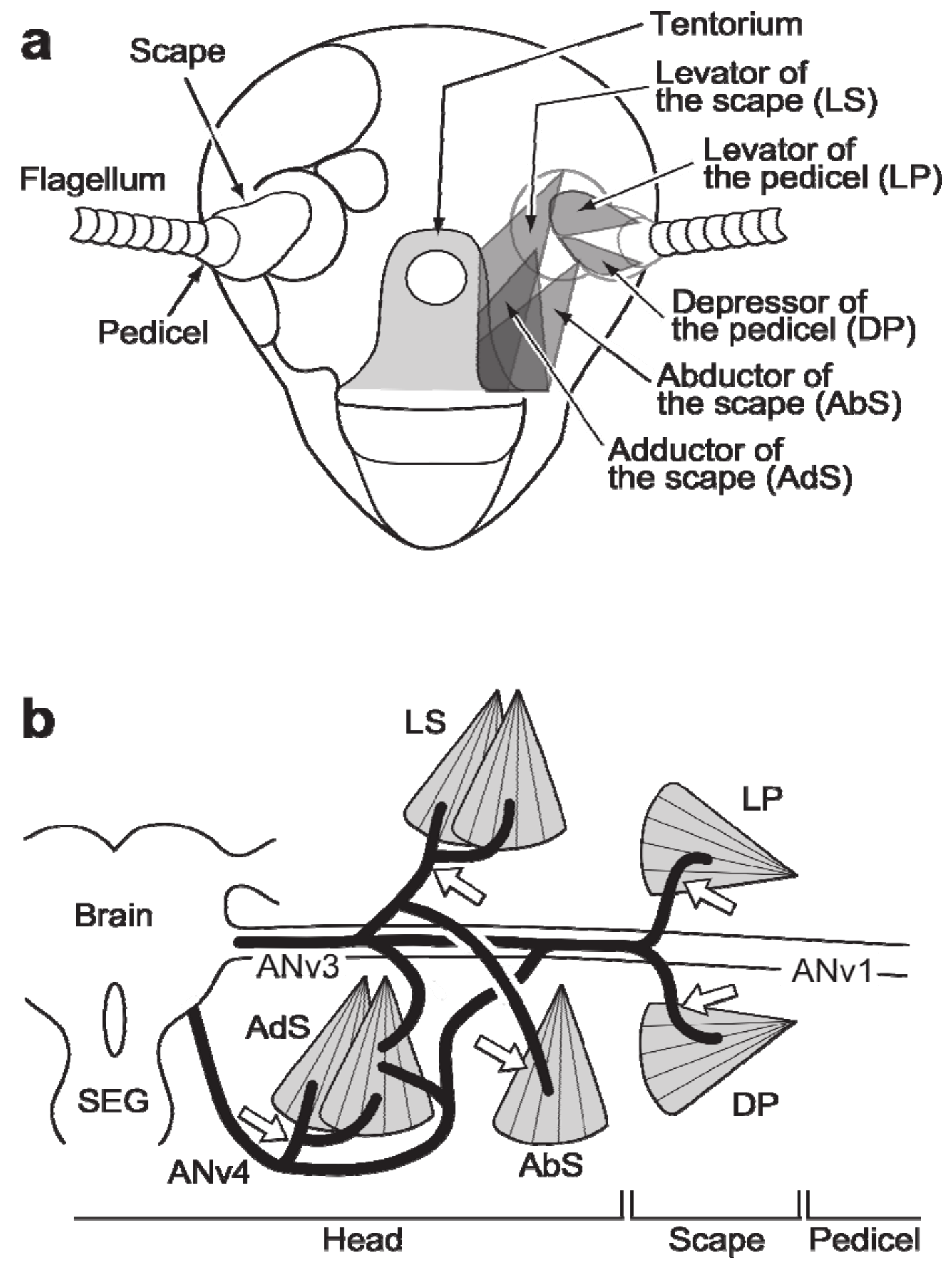
Fig. 3

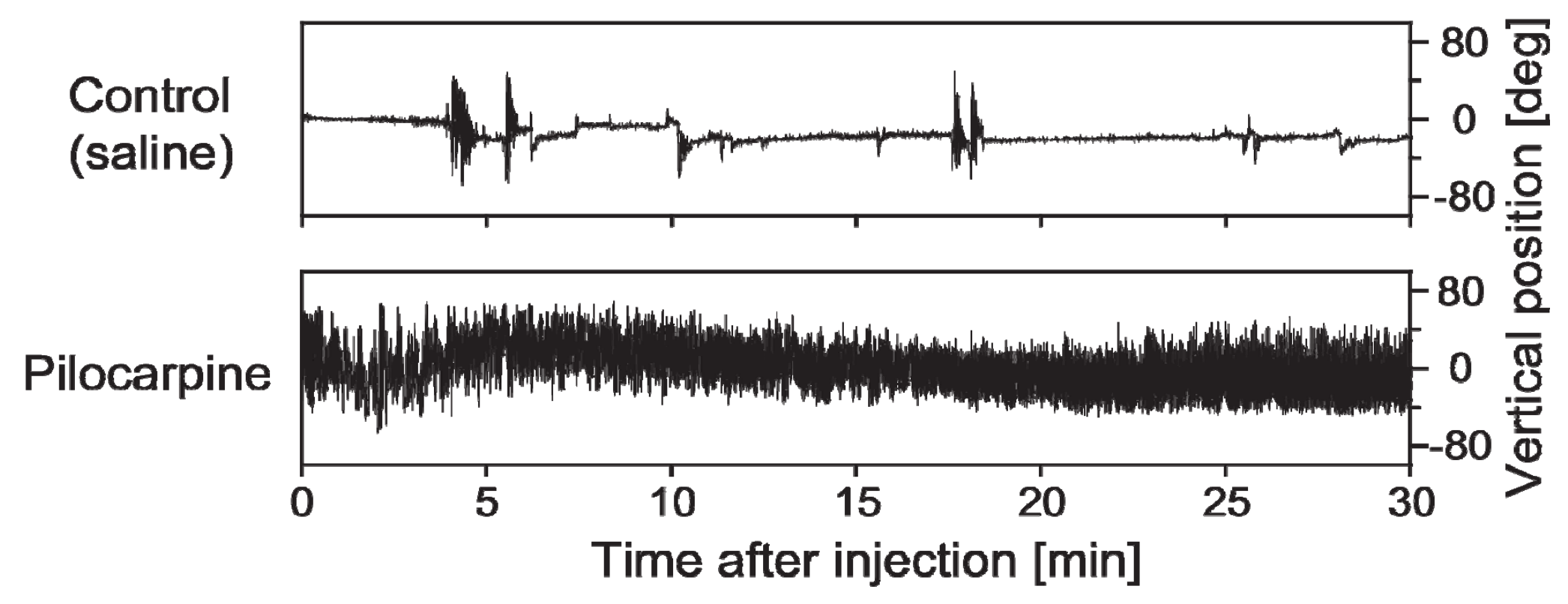


Fig. 4

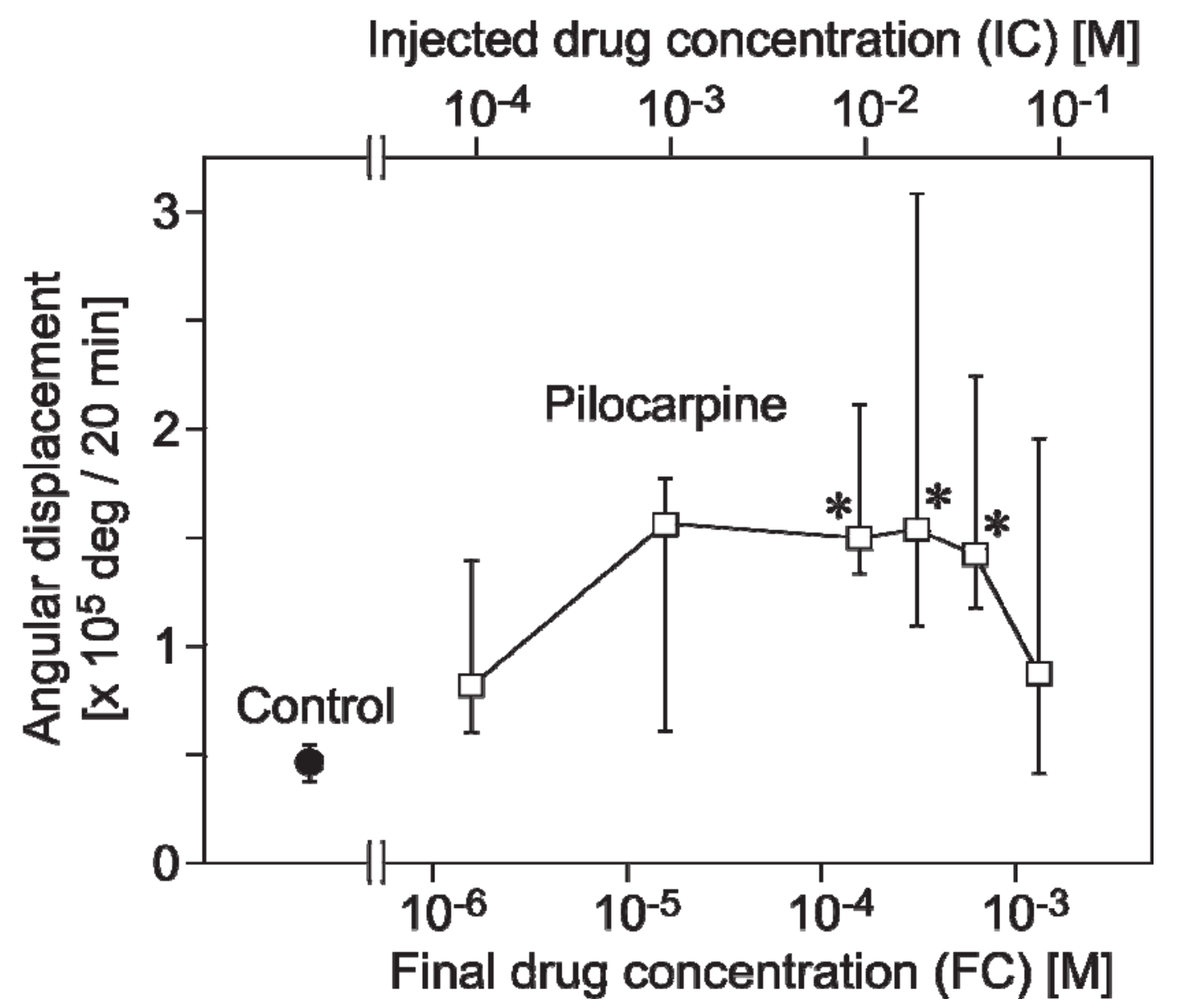


Fig. 5

a

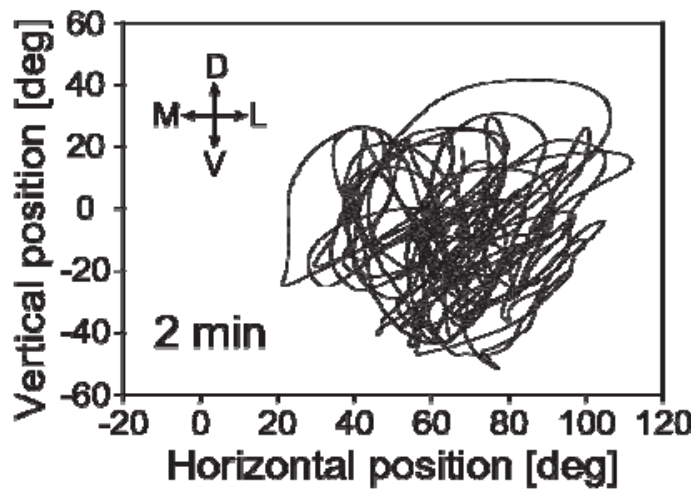

C

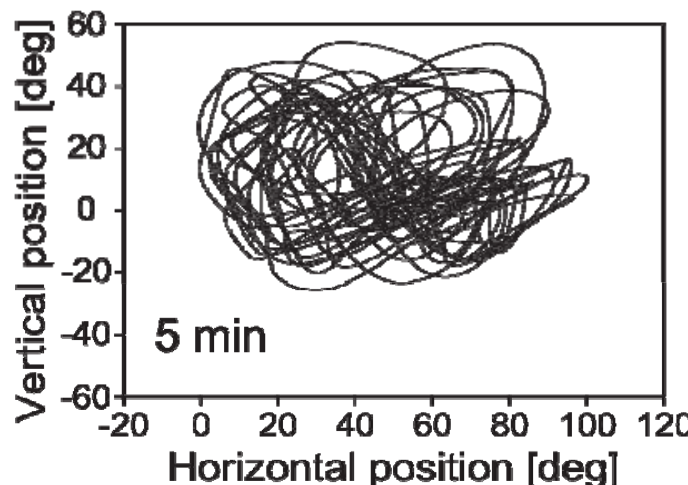

b

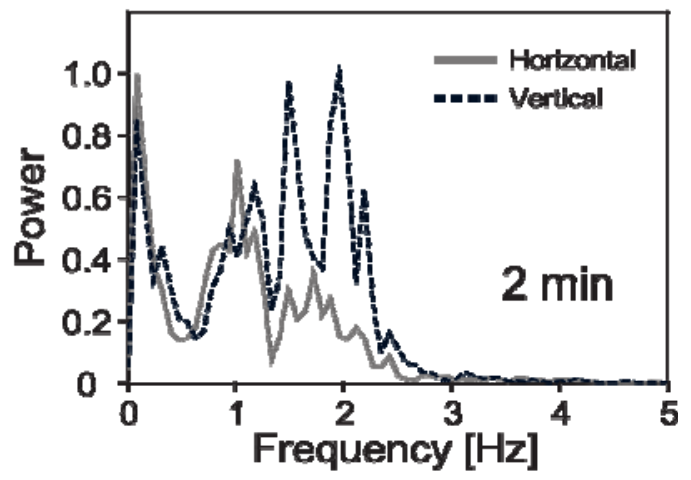

d

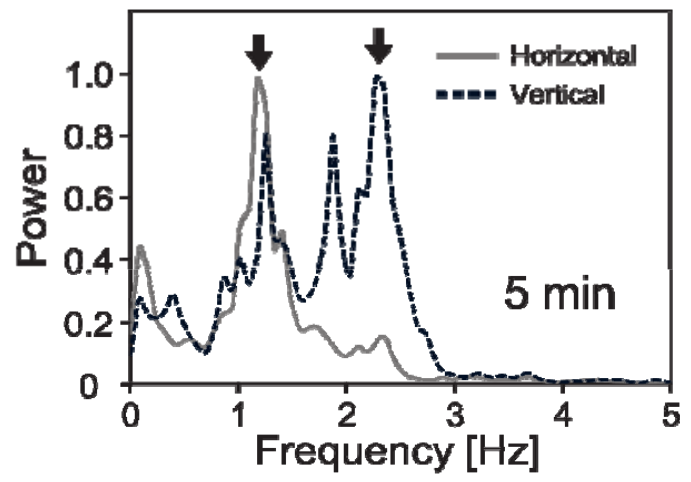

e

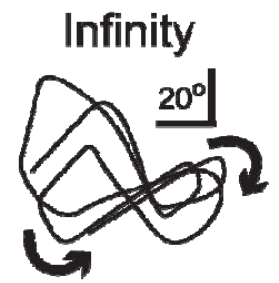<smiles>[Y14][14C]([2H])([2H])[14CH2]</smiles>

Loop
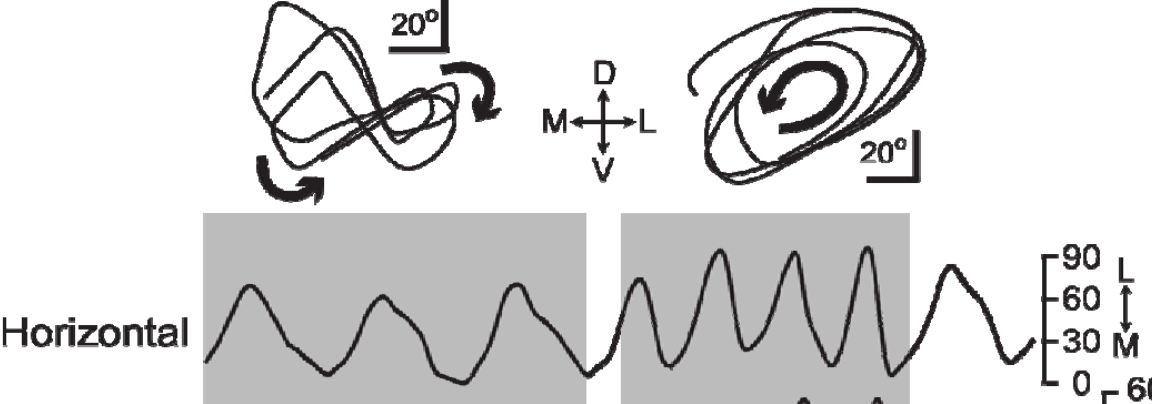

Vertical

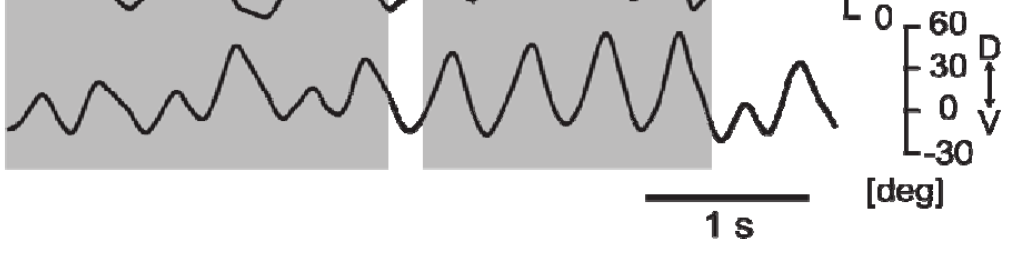


Fig. 6

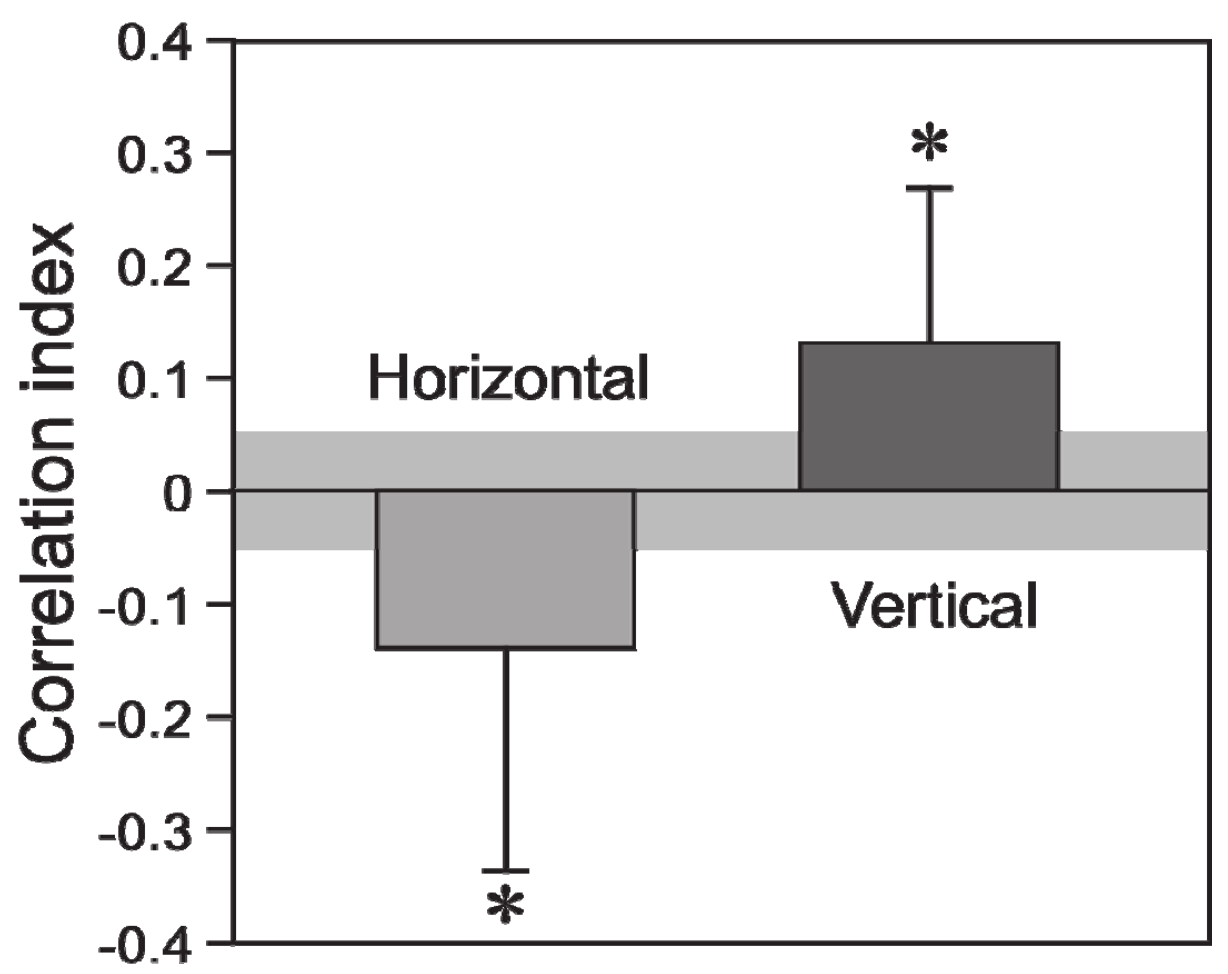


Fig. 7

a

Spontaneous

DP

I IP

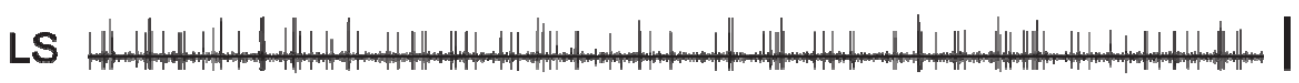

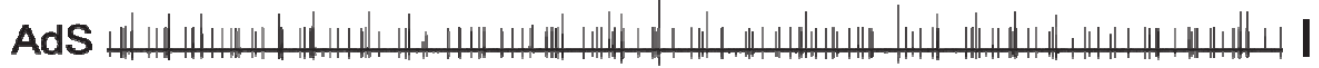

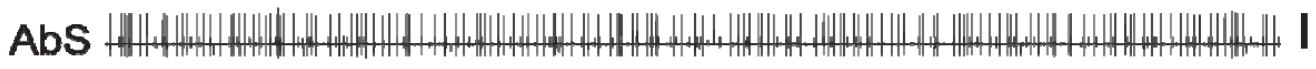

b

Pilocarpine

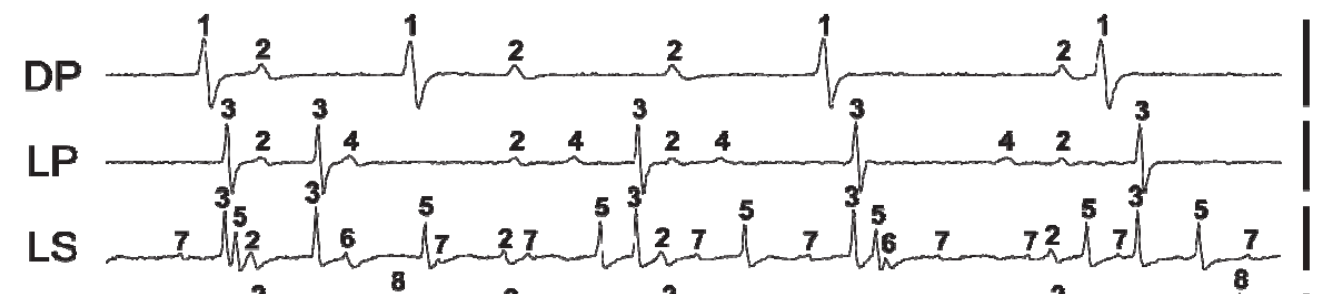

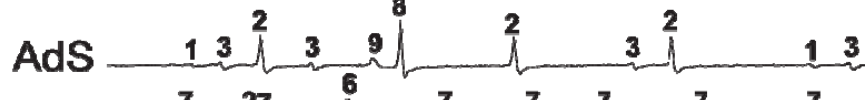

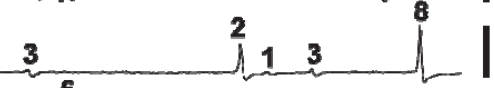

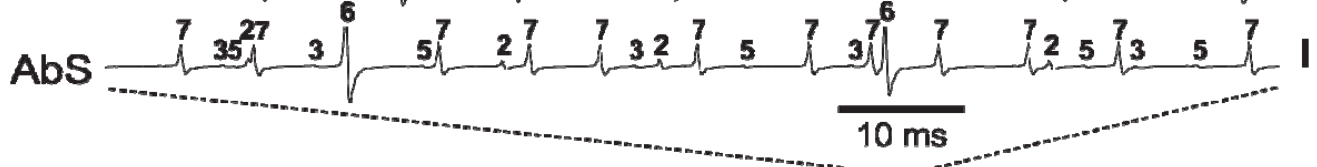

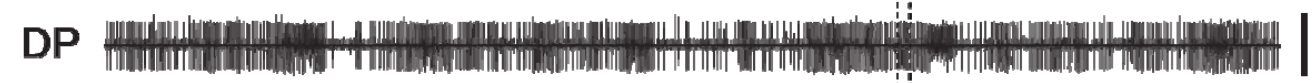

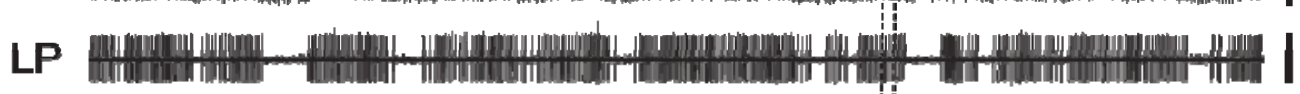

LS

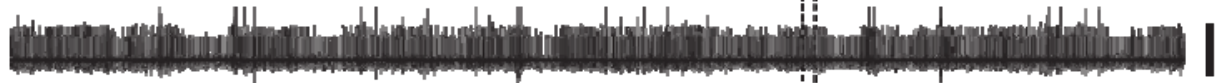

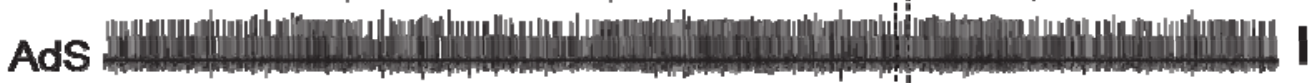

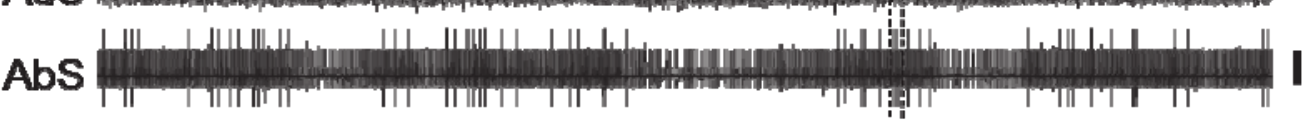

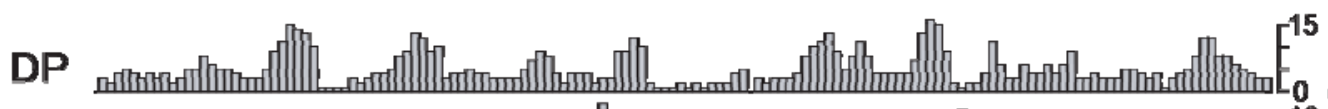

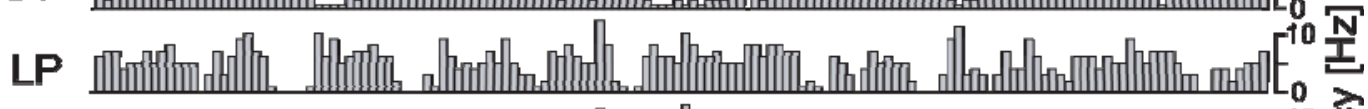

LS

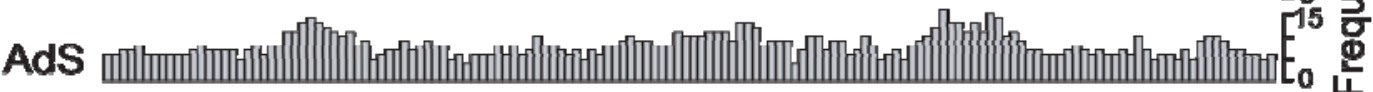

Abs 
Fig. 8

\section{Spontaneous}

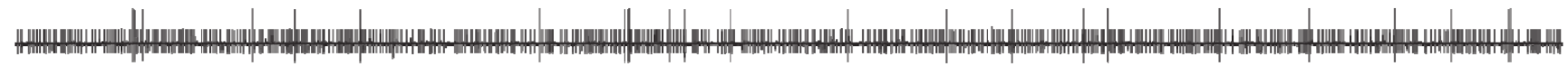

\section{Pilocarpine}

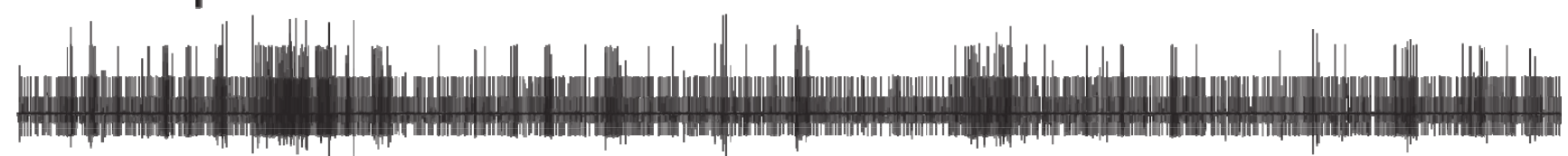

\section{Pilocarpine + atropine}

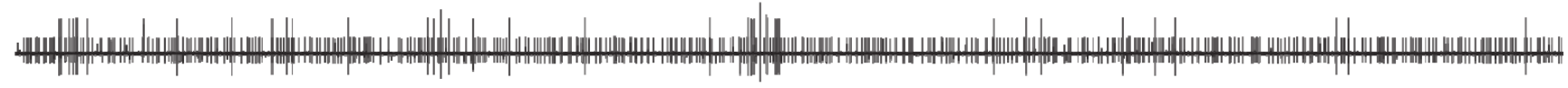

\section{Wash (pilocarpine)}

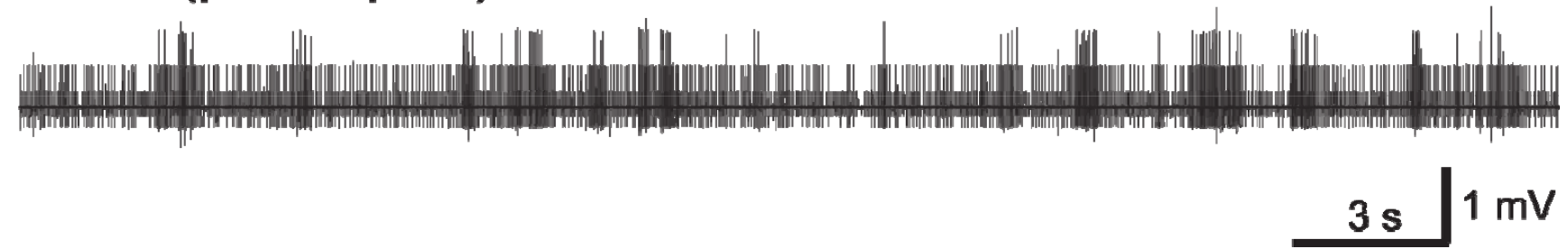


Fig. 9
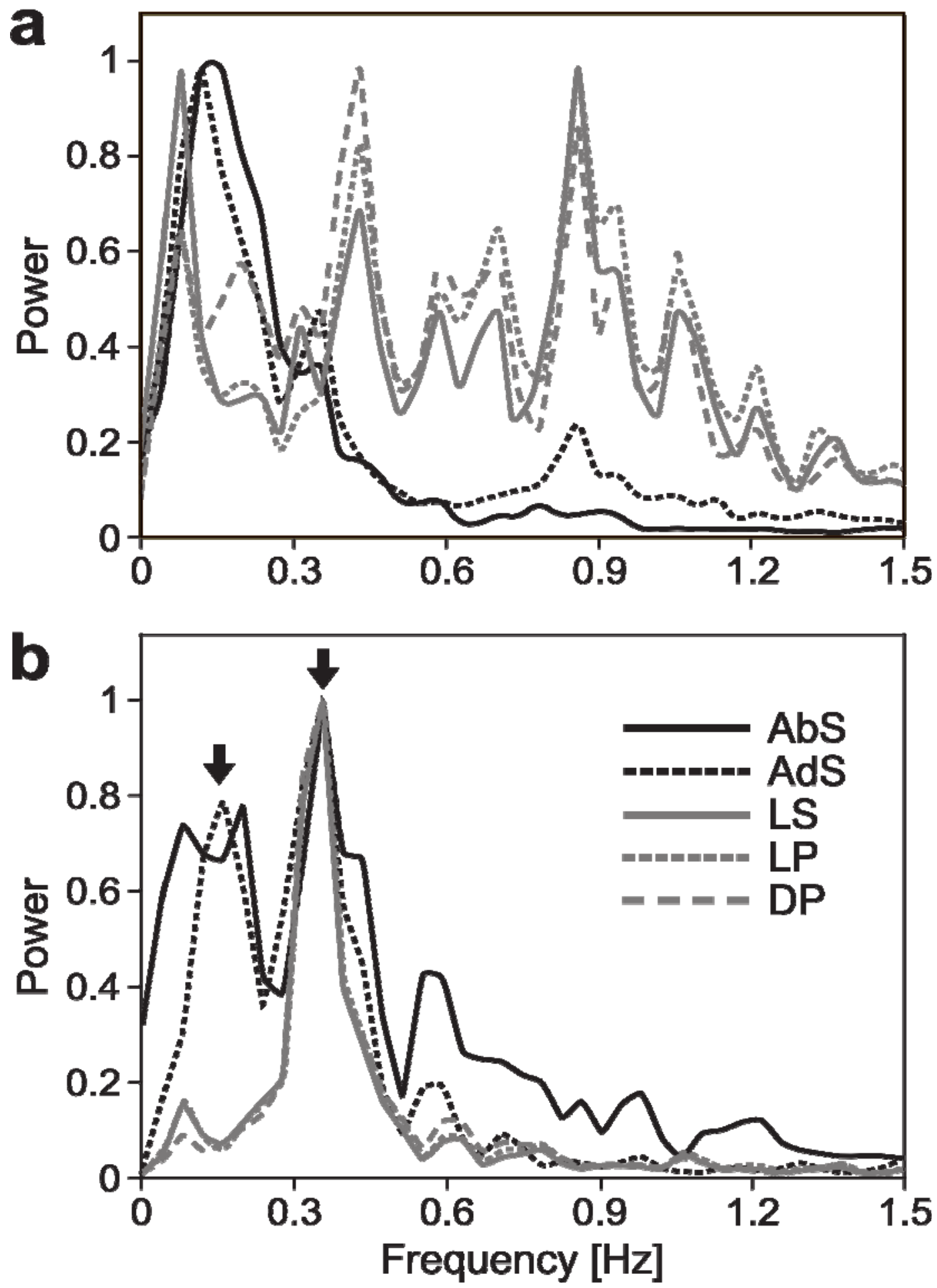
Fig. 10

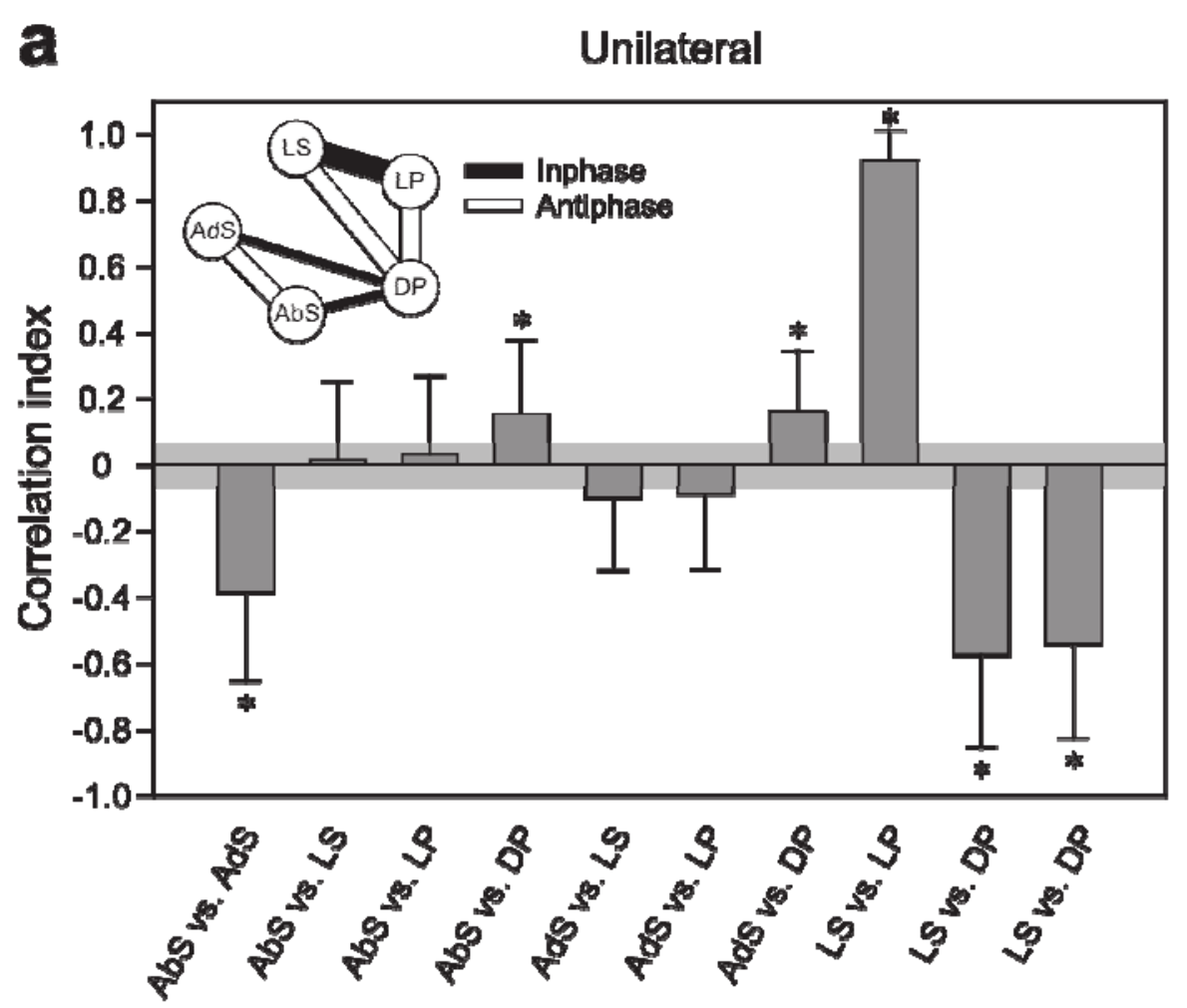

C 1

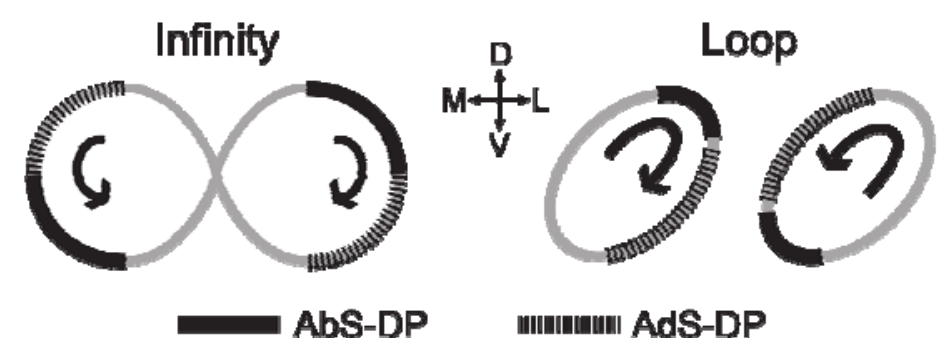

b

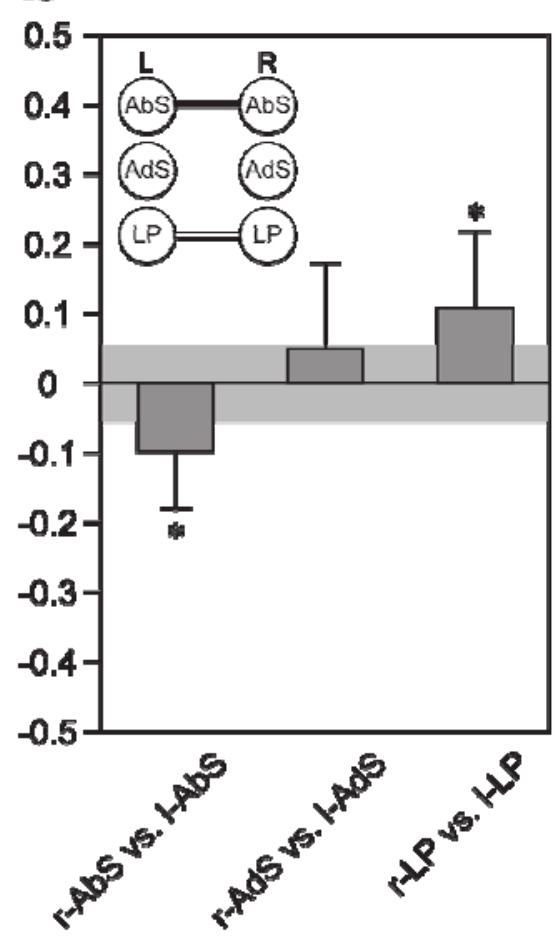

2 Horizontal Vertical

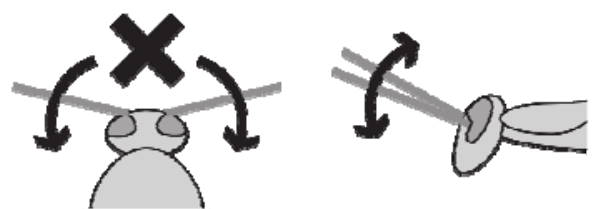

